\title{
Innovation Opportunities: An Overview of Standards and Platforms in the Video Game Industry
}

\author{
Mikael Laakso and Linus Nyman
}

\author{
(6 Man will always use his most advanced technology ") \\ to amuse himself.
}

\author{
David Crane \\ Co-founder, Activision
}

\begin{abstract}
The video game industry offers insights into the significance of standards and platforms. Furthermore, it shows examples of how new entrants can offer innovative services, while reducing their own risk, through bridging the boundaries between standards. Through an exploration of both past and present, this article aims to serve as a primer for understanding, firstly, the technological standards and platforms of the video game industry, and secondly, the recent innovations within the video game industry that have enabled products to be made available across platforms.
\end{abstract}

\section{Introduction}

Over a billion people worldwide play video games (eMarketer, 2013). The revenues of the video game industry (including consoles, hardware and software, online, mobile, and PC games) for 2013 were estimated to total $\$ 93$ billion USD (Gartner, 2013). This revenue is significantly larger than, for instance, the box office revenues of all films released worldwide ( $\$ 35.9$ billion [MPAA, 2013]) and global recorded music sales ( $\$ 15$ billion [IFPI, 2014]) for that same year combined.

What has led the industry to such success? Whereas the foundation of a successful game may be that it is "easy to learn and hard to master" (a phrase attributed to Atari founder Nolan Bushnell), the foundation of the success of the video game industry as a whole, we argue, is standardization - and the innovation that has been able to spring from and around its standards.

Standards have the capability to mitigate both technological and market fragmentation by reducing diversity in solutions where multiple solutions to a specific problem compete. Without the restrictive effect of standards, the potential for both innovation and commercialization is significantly hampered due to a lack of common ground to build upon. Furthermore, standards have served to level the playing field, lower barriers of entry, and allow actors both big and small to compete on more even terms.

The article is structured as follows. First, we offer a brief definition of standards and platforms and how they relate to the video game industry. Then we discuss the emergence of video game platforms and their standards. Finally, we discuss recent as well as upcoming changes and innovations in the video game industry changes that go beyond improvements within a standard to products and services that span multiple standards.

\section{Standards and Platforms}

Technology standards come in many varieties and can emerge through different processes. Thus, succinctly defining the term "standard" poses a challenge. The following definition is used throughout this article:

"A standard is an approved specification of a limited set of solutions to actual or potential matching problems, prepared for the benefits of the party or parties involved, balancing their needs, and intended and expected to be used repeatedly or continuously, during a certain period, by a substantial number of the parties for whom they are meant." (de Vries, 2005) 


\section{An Overview of Standards and Platforms in the Video Game Industry}

Mikael Laakso and Linus Nyman

The spectrum of openness in standards is broad, with potential for complex implications for commercialization (West, 2003). Despite the benefits of technology standardization, the process of settling on a standard can be problematic: it is often, if not always, in tension with technology development. The video game industry has traditionally emphasized having the most advanced graphics and technically impressive presentation. By the time a standard becomes established and widely adopted, it may no longer reflect state-of-the-art hardware or software. Indeed, the video game industry is awash with examples of new innovations and new standards that render previously successful standards all but obsolete. Furthermore, the adoption of a standard - for example, the size of the install base for a specific video game system - influences the availability of software and other complementary products, such as accessories. This network effect creates an environment where a more long-term use of standards and systems is strengthened (Shankar \& Bayus, 2003; Prieger \& $\mathrm{Hu}, 2006)$.

In the video game industry, standards form a key ingredient of platforms (for discussion on the composition of platforms, see, for example: Eisenmann et al., 2008). Indeed, each gaming platform implements a myriad of standards, including those for electric current, connector types, etc. However, for content creators, three central types of standards apply to: i) hardware architectures, ii) operating systems, and iii) software development environments, including both compatibility and compliance guidelines to match the platform holder's requirements. Figure 1 depicts the layered relationships between each of these types of standards. As will be shown throughout the article, innovations in one of these layers affect the others.

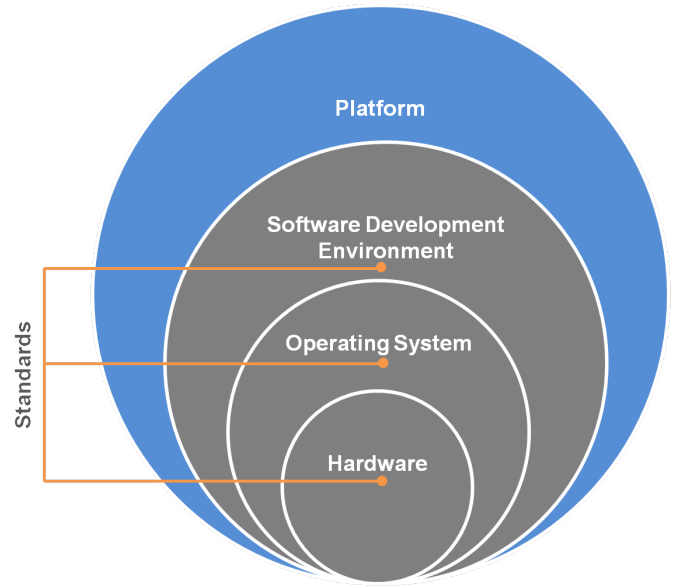

Figure 1. Layers of standards used to create video game platforms
Although standards create platforms, platforms create markets by establishing a common ground between developers and end users. Through platforms, developers know the specific features and functionality of the targeted software and hardware environment, while end users benefit by knowing that adopting a specific platform (e.g., buying a specific video game system) will enable them to access everything developed for it. The adopters of a specific platform define the maximum audience and the content market, while developers produce the content.

\section{The Emergence of Video Game Platforms and Their Early Standards}

In this section, we briefly cover the computer, video arcade, console, and mobile phone platforms, as well as the standards that they are built upon. Such an historical overview allows for viewing the bigger picture of the significance of both the initial lack of certain standards, as well as their later emergence. Additionally, how some platforms, regardless of standardization, have fallen out of public favour due to successful innovations in others.

\section{Computer games}

The earliest computer games of the 1940s and 1950s were housed within massive, custom-built contraptions intended to showcase what computers were capable of (e.g., Donovan, 2010). These computers could only run the one single (and simple) game for which they were built. However, as computers evolved, video games caught the public interest as a form of entertainment. An early favourite, Spacewar!, was released in 1962, making it one of the earliest digital computer games. Computers at the time were not only few and far between, they were also incompatible with other models of computers. Thus, although Spacewar! made famous the PDP-1 (tinyurl.com/mquub) computer for which it was written (for details, see the Computer History $\mathrm{Mu}$ seum: tinyurl.com/y9dgav2), it could not be run on other models of computer without rewriting the source code.

Increased standardization was stimulated during the early 1980 s by the introduction of IBM-compatible computers, all of which could run MS-DOS. This standardization in turn greatly facilitated game development. As a platform, computers are the most flexible when it comes to choice, because there are multiple viable producers for each major platform standard (i.e., hardware, operating system, software development environment) and users can mix and match between them as they see fit. 


\title{
An Overview of Standards and Platforms in the Video Game Industry
}

\author{
Mikael Laakso and Linus Nyman
}

Computers built around x86 processors are currently the dominant hardware architecture, with the main standards for operating systems being Windows, Mac OS, and Linux. For video gaming purposes, Windows remains dominant as the most popular operating system among game developers; however, Mac OS and Linux are seeing an increasing number of high-profile titles released for their platforms as well. Various software development environments are available for all three operating systems, which provide developers with tools to produce compatible software to run on top of the operating system.

\section{Arcade games}

In addition to its significance to computer gaming, Spacewar! also served as the inspiration for the first arcade game, Galaxy Game (tinyurl.com/mzw8qs4), released in 1971. At that time, arcade games were one-off unique creations: big black boxes designed to accept coins in exchange for the opportunity to play one specific game. Each new arcade game system could largely be designed from scratch, without much concern regarding uniformity in either hardware or software across the line of existing arcade games. The business model was similarly designed around delivering one-off, bite-sized experiences (i.e., insert coin, play the game) without requiring that the consumer buy or invest in the system outright, lessening the need to establish conformity among arcade systems.

Though it took longer than in other video game platforms, arcade games also saw the introduction of influential standards. The Japan Amusement Machinery Manufacturer's Association standard (JAMMA; tinyurl .com/cv88dpk), developed in 1985 by an industry consortium of arcade game developers, made it possible to create more modular hardware, with arcade games programmed on detachable game cartridges. These cartridges, hidden inside the arcade cabinet itself, enabled the arcade owner or operator to change a game while keeping the same arcade cabinet. The same hardware could thus be used to run different software, lowering manufacturing costs for the arcade boards and reducing the need for expensive logistics for switching cabinets around different locations. However, advances in both home computers as well as consoles would soon usher in the end of the golden age of the video arcade. Throughout the rest of the article, focus is placed on other platforms that still have a strong presence today.

\section{Consoles}

Video game consoles for home use made their debut in the late 1960s (TIME, 2014). The first devices came with only one, or sometimes a few, built-in games, without any possibility of running additional software code. As with arcade games, this one-off nature of the product lessened the need for any strict standards to be implemented, given that the systems were closed from further hardware or software expansions.

A significant development for home consoles came with the release of the first systems for which one could program games for later individual purchase. This advancement also introduced a new source of income and fundamentally different business model for platform holders. It was the Atari 2600 (tinyurl.com/odlwg), released in 1977, that popularized the use of game cartridges. During its heyday, another breakthrough happened in the dynamics of game development for game consoles: third-party development (Barton \& Loguidice, 2008). In the past, it had mostly been console manufacturers that created and published games for their own platforms, but third-party development for the Atari 2600 thrived, despite initial legal efforts from Atari to thwart the sale of such games (e.g., Atari \& Tengen vs. Nintendo, 1992). Though the relationship between platform holder and third-party developer can usually be assumed to be symbiotic, there have been multiple attempts by software developers to circumvent software licensing fees and potential authentication methods by reverse engineering compatible cartridges (see, for example: Linhoff, 2004).

Although the hardware and software standardization involved in cartridges allowed for compatibility within a console, there was also an effort at creating a crosscompany platform standard for consoles. This effort came in the form of the short-lived 3DO project (tinyurl.com/on7gb2n), released in 1993. 3DO was a consortium owned by several manufacturers, allowing anyone within the consortium the right to manufacture a 3DOcompatible console as long as they paid a license fee. This approach can be compared to, for instance, VHS and DVD standards, where anyone could produce compatible hardware.

The major hardware release cycles in the video game industry are commonly referred to as generations, with the latest console releases (e.g., Xbox One: tinyurl.com/n72ba7c; PlayStation 4: tinyurl.com/nfs5yuq; and Wii U: tinyurl.com/8zgpyz3) being Generation 8. A shift from one generation to the next has often left software from previous generations incompatible with the new platform's hardware, meaning that the software library is often thin during the initial launch period. Backwards compatibility has been suggested to facilitate the suc- 


\title{
An Overview of Standards and Platforms in the Video Game Industry
}

\author{
Mikael Laakso and Linus Nyman
}

cess of the system (Kramer \& de Vries, 2009). However, having to potentially design the new system around old standards for hardware architecture and media format might constrain the degree to which the system can introduce state-of-the-art features.

\section{Mobile phones}

Standards played a critical role in the evolution of mobile phones and the subsequent smartphones and tablets. Though appearing several decades later than consoles, mobile phones have in many respects echoed their evolution. Mobile phones, like consoles, initially came with a limited amount of built-in games and without any means of expansion. It was not until the first smartphones that it became possible for users to install additional software on handsets. However, actual widespread adoption of software expansion had to wait for several years due to technological fragmentation and a lack of viable methods for both distribution and payment. Before the App Store, Google Play, and Windows Phone store, the only way to install software on smartphones was to side-load the installation files by first downloading them from the web, potentially paying for them, and then executing the files through a file manager. By standardizing the means of acquiring games and apps, a new - and hugely profitable - market was created.

Mobile phones have evolved from dedicated appliances into small powerful computers. Currently, smartphones and tablets are almost exclusively built around architectures implementing ARM processors on the hardware level. On top of this common ground, differentiation is achieved through hardware configurations and operating systems (e.g., Apple's iOS, Google's Android, Microsoft's Windows Phone). Each of these platforms has their own development environments and storefronts for distributing software. Apple controls the entire stack: hardware, operating system, and software development environment. Microsoft and Android provide the operating system and software development environment; although they do not provide the hardware, they offer guidelines for hardware manufacturers to abide by for compatibility.

With the move to mobile devices (i.e., phones and tablets), many significant changes occurred in the gaming industry. One such change was improved access as games became more readily available on non-dedicated gaming devices. Many, including those that had not previously engaged with games, now carried with them a device on which they could play games. By way of example, in 2013 more than half of US mobile phone owners (125.9 million people) were estimated to have played games on their phone (eMarketer, 2013). The increase in gamers, as well as potential gamers, resulted in the rise in popularity of casual games (e.g., puzzle games and match-three games). Significant changes also occurred on the business model front. Though income initially was generated from selling the games, other business models soon emerged: generating income through ads, through offering in-app purchases, or a combination of the two.

Not surprisingly, developers large and small are responding to the increased demand for mobile games: over 220,000 games were released on Apple's iOS alone during the first four years since its launch, making games the largest category of application overall (Pocket Gamer, 2012). The most financially successful mobile games generate incomes in the millions of dollars per day (Strauss, 2013). However, the rise of the mobile gaming industry has been a significant generator of revenue for platform holders as well, as they take a cut of all sales.

\section{Standardization patterns}

As can be seen even from so brief an overview of the evolution of the video game industry, wherever a new form of video game was developed, standardization inevitably followed. Indeed, as games moved out of the arcades and into our living rooms, and more recently onto our mobile devices, the significance as well as proliferation of standards has grown considerably.

Although the triple layer of hardware, operating system, and software development framework is applicable across gaming platforms, there are differences in its implementation. Video arcades are perhaps most notable in this sense in that they did not commonly include an operating system. Modern consoles bundle the entire stack into one product, whereas computers and mobile phones (to varying degrees) allow for variability in the stack by either hardware manufacturers or users themselves.

Over time, development and standards have evolved to become more high-level. Where initially there was very little separating the programmer from the hardware, today development frameworks and other middleware facilitate development by providing toolsets that let programmers focus on creating content rather than having to learn and manage the intricacies of the hardware architecture. High-level development also facilitates porting, meaning releasing games across multiple platforms. A further significant development is that 


\title{
An Overview of Standards and Platforms in the Video Game Industry
}

\author{
Mikael Laakso and Linus Nyman
}

game developers have become more involved in the standardization process. Whereas the console or platform holder previously dictated standards, they now commonly evolve as more of a joint effort among several stakeholders.

\section{Beyond Standards}

While innovations such as the App Store and Google Play have made accessing mobile device software easier for consumers, other new entrants have sought to bridge both computer hardware and operating system platform divides. Good old Games (gog.com) offers old games rewritten to work across a broader spectrum of hardware and operating systems. Game publishers have similarly launched their own storefronts, or platforms, combining elements such as digital distribution, digital rights management (DRM), multiplayer, and social networking. The three main storefronts are: Electronic Arts' Origin (origin.com), Ubisoft's Uplay (uplay .ubi.com), and Valve's Steam (store.steampowered.com). These storefronts, commonly available for several different operating systems, provide online purchasing of, and subsequent access to, games by both the publishers themselves and by other developers. However, because these storefronts are not a platform defined at the hardware level, they do not guarantee that the customer's hardware is compatible with the game requirements. Steam is arguably the most successful such storefront, and has to a great extent managed to unify a fragmented computer market despite the diversity in hardware specifications and non-standardized DRM practices across publishers.

Browser-based games also span hardware and operating system divides, allowing access through any platform (not just computers) that offers access to a standard web browser, regardless of hardware or operating system. HTML5 has enabled advanced native web programming functionality, rendering the use of external browser plugins, such as Adobe Flash, optional. Similarly, massively multiplayer online games (MMOGs; tinyurl.com/fzbyv) commonly offer separate game clients for different operating systems, thus allowing common access to a game regardless of operating system.

Some of the most significant platform spanning has occurred in the area of software development environments. Traditionally, developers had to choose, before starting a project, for which platform they wanted to develop their game, and they were subsequently more or less locked in to that platform. Innovations in the in- dustry have granted developers substantial freedom from such limitations. Game development tools such as the Unreal Engine (unrealengine.com) and Unity (unity3d.com) have enabled game developers to work largely independent of platform considerations. These tools make it possible to develop a game first, and then publish to one or multiple platforms upon completion.

In the not-so-distant future, gaming may become almost completely detached from platforms and hardware considerations due to platform spanning on the side of end users. Online video game streaming services, currently pioneered by Onlive (games.onlive.com) and Playstation Now (tinyurl.com/mbbqav5), work like Software as a Service (tinyurl.com/2j3d5z): the actual game being played runs on a remote server. The gamer's controller inputs (i.e., what they want to do in the game) are transmitted to the server, and the video and audio feed of the game (i.e., what then happens in the game) are transmitted back to the gamer's screen. This architecture results in minimal hardware requirements on the user's end, while the back-end at the service provider can be upgraded without the user making any new hardware purchases. However, low-latency, highspeed broadband access is essential for this approach to become commonplace, something that is currently not ubiquitous on a global scale.

During the last two decades, there has been a substantial performance gap between the technological capabilities of stationary versus mobile devices, a gap that has been closing as technology has evolved. Shigeru Miyamoto, the top game designer at Nintendo, recently stated that they are considering the unification of their home and portable console hardware architectures to facilitate more efficient game development (Kaiser, 2014). This unification would mark a historical change in their hardware and software development strategy, which has been split in two distinct components since the 1980s.

\section{Conclusions}

The early years of the video game industry were a time of almost exclusive in-house development, with little in the way of standards either within or across platforms. This early phase was followed by a gradual standardization, which opened up the gaming industry to thirdparty development. More recent innovations have enabled the spanning of platforms, making games more easily available across several platforms, as well as making it easier to develop games for multiple standards. 


\section{An Overview of Standards and Platforms in the Video Game Industry}

Mikael Laakso and Linus Nyman

The lines between platforms are blurring and unification is happening on many fronts due to the proliferation and advancement of standards, some becoming so integrated that their implementation and use is transparent on the surface. This process is happening both on the side of game development as well as for players.

The gaming industry offers insights into the importance of standardization, but goes beyond that to show the opportunities that exist for those who manage to offer products or solutions either on a higher level of standard, or indeed that can span multiple standards or platforms.

Even an evolving industry can find itself become nearobsolete. Advances and innovations within video arcades were rendered all but irrelevant by advances and innovations in the home computer and console platforms. Now, some believe gaming computers and consoles may be going the way of the arcade due to advances in handheld devices, offering ease of access as well as promising to turn tablets into de-facto gaming computers for the home through streaming services.

As shown through the history of the video game industry, standards traditionally mitigate both technological and market fragmentation. Standards have been used to create technological platforms on to which content creation and commerce can be conducted. However, these platforms have mostly been isolated due to a lack of cross-compatibility, which segments the market for both software developers and end users. It is only recently that platform spanners have emerged for both software developers and end-users, creating bridges between platforms. The hierarchy for the chain of relationships is depicted in Figure 2.

Where an individual platform can be left abandoned by new technological advances being introduced and the market migrating to more modern options, innovative platform spanners do not rely on the success of any single platform. This flexibility benefits all major stakeholders in the videogames industry and facilitates a more inclusive market space where more content is made available on more devices than ever before.

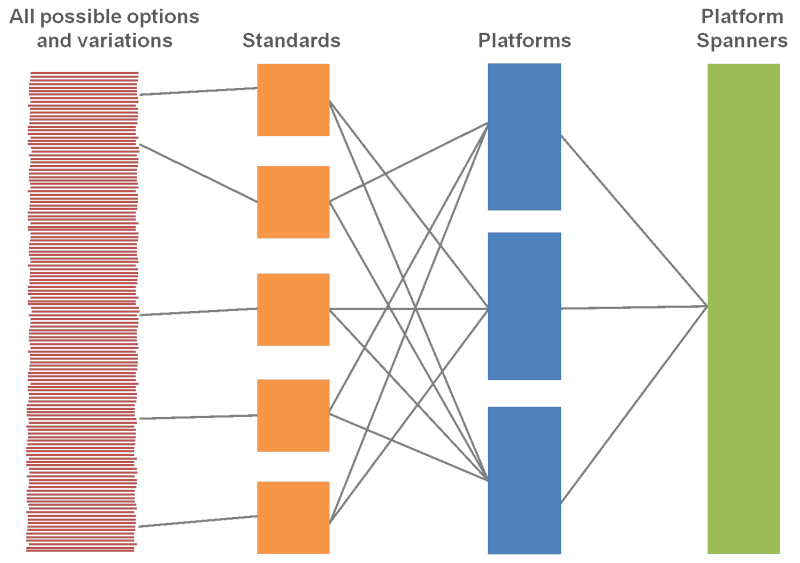

Figure 2. Innovative platform spanners unify standards and platforms with potential benefits to both developers and consumers

\section{Recommended Reading}

- Replay: The History of Video Games

(Donovan, 2010; tinyurl.com/bwu4qyz)

- "For Amusement Only: The Life and Death of the American Arcade"

(June, 2013; tinyurl.com/aawzxev)

- "Industry Life-Cycle Theory in the Cultural Domain: Dynamics of the Games Industry"

(Peltoniemi, 2009; tinyurl.com/nx27wy9)

- "Structure and Competition in the US Home Video Game Industry"

(Williams, 2002; tinyurl.com/lh223ys)

- "Entry into Platform-Based Markets"

(Zhu \& Iansiti, 2011; tinyurl.com/p5c22uu) 


\section{An Overview of Standards and Platforms in the Video Game Industry}

Mikael Laakso and Linus Nyman

\section{About the Authors}

Mikael Laakso is a postdoctoral researcher at the Hanken School of Economics in Helsinki, Finland. He has published research on open access in scientific publishing and standardization of construction IT. Mikael has a Doctoral and Master's degree in Information Systems Science from the Hanken School of Economics.

Linus Nyman is a doctoral researcher at the Hanken School of Economics in Helsinki, Finland, where he has recently submitted his $\mathrm{PhD}$ on code forking in open source software. Linus has a Master's degree in Economics from the Hanken School of Economics.

\section{References}

Atari \& Tengen vs. Nintendo, 1992. United States Court of Appeals for the Federal Circuit, 91-1293.

Barton, M., \& Loguidice, B. 2008. A History of Gaming Platforms: Atari 2600 Video Computer System/VCS. Gamasutra. July 1, 2014: http://www.gamasutra.com/view/feature/131956/a_history_of_gaming_platforms_.php

Donovan, T. 2010. Replay: The History of Video Games. Hove, UK: Yellow Ant Media.

de Vries, H. 2005. IT Standards Typology. In: Jakobs K. (Ed.) Advanced Topics in Information Technology Standards and Standardization Research, 1:11-36. Hershey, PA: Idea Group Publishing.

Eisenmann, T. R., Parker, G., \& Van Alstyne, M. W. 2008. Opening Platforms: How, When and Why? Harvard Business School Entrepreneurial Management Working Paper No. 09-030. Cambridge, MA: Harvard Business School.

http://dx.doi.org/10.2139/ssrn.1264012

MPAA. 2013. Theatrical Market Statistics, 2013. Motion Picture Association of America. July 1, 2014: http://www.mpaa.org/wp-content/uploads/2014/03/MPAA-Theatrical-Market-Statistics-2013_032514-v2.pdf
eMarketer. 2013. Half of US Mobile Users to Play Games in 2013. eMarketer. July 1, 2014:

http://www.emarketer.com/Article/Half-of-US-Mobile-Users-PlayGames-2013/1009928

Gartner. 2013. Gartner Says Worldwide Video Game Market to Total \$93 Billion in 2013. Press Release: October 29, 2013.

http://www.gartner.com/newsroom/id/2614915

IFPI. 2014. Key Statistics. International Federation of the Phonographic Industry. July 1, 2014:

http://www.ifpi.org/global-statistics.php

Kaiser, T. 2014. Nintendo's Top Designer: We're Looking to Unify Mobile, Console Games. DailyTech. July 1, 2014:

http://www.dailytech.com/Nintendos+Top+Designer+Were+Looking+to+Unify+Mobile+Console+Games/article36107c.htm

Linhoff, J. 2004. Video Games and Reverse Engineering: Before and After the Digital Millennium Copyright Act. Journal on Telecommunications \& High Technology Law, 3: 209-237.

Kramer, J., \& de Vries, H.J. 2009. Impact of Backwards Compatibility on Standard Dominance - The Case of Game Consoles. Proceedings of the 2009 European Academy of Standardisation (EURAS) Conference: $149-160$.

Pocket Gamer. 2012. App Store Metrics, September 2012. Pocket Gamer. July 1, 2014: http://www.pocketgamer.biz/metrics/app-store/

Shankar, V., \& Bayus, B. L. 2003. Network Effects and Competition: An Empirical Analysis of the Home Video Game Industry. Strategic Management Journal, 24(4), 375-384.

http://dx.doi.org/10.1002/smj.296

Strauss, K. 2013. The \$2.4 Million-Per-Day Company: Supercell. Forbes. July 1, 2014:

http://www.forbes.com/sites/karstenstrauss/2013/04/18/the-2-4million-per-day-company-supercell/

Spil Games. 2013. State of Online Gaming Report. Spil Games. July 1, 2014:

http://www.spilgames.com/state-online-gaming-2013-2/

TIME. 2014. A History of Video Game Consoles. TIME. July 1, 2014: http://content.time.com/time/interactive/0,31813,2029221,00.html

Prieger, J. E., \& Hu, W. M. 2006. An Empirical Analysis of Indirect Network Effects in the Home Video Game Market. Working Paper No. 06-25. New York: NET Institute. http://dx.doi.org/10.2139/ssrn.941223

West, J. 2003. How Open Is Open Enough? Research Policy, 32(7): 1259-1285. http://dx.doi.org/10.1016/S0048-7333(03)00052-0

Citation: Laasko, M., \& Nyman, L. 2014. Innovation Opportunities: An Overview of Standards and Platforms in the Video Game Industry. Technology Innovation Management Review, 4(7): 15-21. http://timreview.ca/article/808

Keywords: video game industry, standards, platforms, innovation, computer games, arcade games, console games, mobile games 